June 23, 2011

\title{
Integral points of a modular curve of level 11
}

\author{
by René Schoof and Nikos Tzanakis
}

\begin{abstract}
Using lower bounds for linear forms in elliptic logarithms we determine the integral points of the modular curve associated to the normalizer of a non-split Cartan group of level 11. As an application we obtain a new solution of the class number one problem for complex quadratic fields.
\end{abstract}

\section{Introduction.}

Let $E$ be the elliptic curve given by the Weierstrass equation

$$
Y^{2}+11 Y=X^{3}+11 X^{2}+33 X
$$

This is the curve 121B1 in Cremona's table [4, p.121]. By [4, p.256] the group of rational points of $E$ is the infinite cyclic group generated by the point $(0,0)$. The main result of this paper is the following.

Theorem 1.1. There are precisely seven rational points $(x, y)$ on $E$ for which

$$
\frac{x}{x y-11}
$$

is integral. They are $(0,0),(0,-11),(-2,-5),(-2,-6),(-6,-2),(-11 / 4,-33 / 8)$ and the point at infinity.

Let $X_{n s}(11)$ denote the modular curve associated to the normalizer of a non-split Cartan subgroup of level 11; see [10, Appendix]. This curve has genus 1, is defined over $\mathbf{Q}$ and parametrizes elliptic curves with a certain level 11 structure. The interest of Theorem 1.1 lies in the fact that $X_{n s}(11)$ is isomorphic over $\mathbf{Q}$ to the curve $E$ and that rational points $(x, y)$ on $E$ for which $x /(x y-11)$ is integral, correspond precisely to integral points on $X_{n s}(11)$, i.e., to rational points for which the parametrized elliptic curve has its $j$-invariant in $\mathbf{Z}$. We prove this in section 4 and hence obtain the following corollary of Theorem 1.1.

2000 Mathematics Subject Classification 11DXX, 11F03, 11G05, 11J86, 11R29 
Theorem 1.2. There are precisely seven integral points on the modular curve $X_{n s}(11)$.

As explained by J-P. Serre in the Appendix of [10], every imaginary quadratic order $R$ of class number 1 in which the prime 11 is inert, gives rise to an integral point on $X_{n s}(11)$. The elliptic curve that is parametrized by this point admits complex multiplication (CM) by $R$. Since 11 is inert in the quadratic orders of discriminant $-3,-4,-12,-16,-27$, -67 and -163 , all of which have class number 1 , the seven integral points of Theorem 1.1 are accounted for by these CM curves. See section 4 for the precise correspondence.

If the class number of the imaginary quadratic order of discriminant $\Delta$ is 1 , then the prime 11 is inert in it whenever $|\Delta|>44$. Therefore the fact that there are no other integral points on $X_{n s}(11)$ gives an independent proof of the Baker-Heegner-Stark theorem $[1,7,13]$ : the only imaginary quadratic orders with class number 1 are the ones with discriminant equal to one of $-3,-4,-7,-8,-11,-12,-16,-19,-27,-28,-43$, -67 and -163 .

Our proof exploits effective lower bounds for linear forms in elliptic logarithms [5]. In this respect it differs from earlier work by M. Kenku [8] and B. Baran [2], who exploit modular curves of level 7 and 9 respectively. In both cases the curves involved have genus 0 and the problem is reduced to a cubic Thue equation, which is solved by Skolem's method.

The paper is organized as follows. In section 2 we prove two inequalities. These are used in section 3, where we apply the method of linear forms in elliptic logarithms and prove Theorem 1.1. In section 4 we explain the relation with the modular curve of level 11 and prove Theorem 1.2. All calculations can be checked easily and quickly by means of the PARI software package.

\section{Two inequalities.}

In this section we prove two inequalities concerning the elliptic curve $E$ given by the Weierstrass equation $Y^{2}+11 Y=X^{3}+11 X^{2}+33 X$. The first inequality regards a property of the group $E(\mathbf{R})$ of real points of $E$, while the second is concerned with heights of points in the group $E(\mathbf{Q})$ of rational points.

Let $t$ be the function on $E$ given by

$$
t=Y-\frac{11}{X}
$$

It has simple poles at the points $(0,0)$ and $(0,-11)$ and a pole of order 3 at infinity. Its zero locus consists of five distinct points, which we call the cusps of $E$ because under the isomorphism of section 4 they correspond to the cusps of the modular curve $X_{n s}(11)$. The $x$-coordinates of the cusps are the zeroes of the polynomial $X^{5}+11 X^{4}+33 X^{3}-121 X-121$. In particular, they are all real. It follows that the cusps are contained in the group $E(\mathbf{R})$. The curve $E$ has only one connected component over $\mathbf{R}$, so that $E(\mathbf{R})$ is homeomorphic to a circle.

Writing $F(X, Y)=Y^{2}+11 Y-X^{3}-11 X^{2}-33 X$, we define the function $g$ by

$$
g=\operatorname{det}\left(\begin{array}{ll}
\frac{\partial t}{\partial X} & \frac{\partial t}{\partial Y} \\
\frac{\partial F}{\partial X} & \frac{\partial F}{\partial Y}
\end{array}\right)=3 X^{2}+22 X+33+\frac{11(2 Y+11)}{X^{2}} .
$$

It has poles of order 2 at $(0,0)$ and $(0,-11)$ and a pole of order 4 at infinity. It has eight zeroes on $E$, four of which are real. 
Lemma 2.1. Let $U$ be the subset of $E(\mathbf{R})$ given by

$$
U=\left\{P \in E(\mathbf{R}):|t(P)|<\frac{1}{20}\right\}
$$

Then

(a) the set $U$ is the disjoint union of five open intervals, each containing precisely one cusp;

(b) the function $g$ satisfies $|g(P)| \geq 1$ for every $P \in U$.

Proof. In the proof all values of the functions $g$ and $t$ are given with an accuracy of two decimals only. The values of $t$ in the four real zeroes of $g$ on $E(\mathbf{R})$ are equal to -7.39 , $0.63,-0.16$ and -23.06 respectively. Since the absolute value of each of these numbers exceeds $\frac{1}{20}$, the function $g$ has no zeroes in $U$ and hence Lagrange's multiplier method ensures that $t$ assumes no extremal values in $U$. It follows that $U$ is a union of open intervals $I$, each of which contains at most one zero of $t$. If $t$ were not to vanish on an interval $I$, then its values on the boundary points of $I$ would either be both equal to $+\frac{1}{20}$ or to $-\frac{1}{20}$. This is impossible as $t$ assumes no extremal values on $I$. This shows that $t$ has at least one zero in $I$ and (a) follows.

To prove $(b)$, note that the values of $g$ in the five cusps are $9.75,-1.78,1.39,-3.79$ and 159.43 respectively. Therefore $|g(P)| \geq 1$ for all points $P$ in a sufficiently small neigborhood of the cusps. We need to show that $U$ is such a neighborhood. We saw in the proof of part (a) that $g$ has no zeroes in $U$. Lagrange's multiplier method shows that $g$ assumes its extremal values in the zeroes of the function

$$
\operatorname{det}\left(\begin{array}{ll}
\frac{\partial g}{\partial x} & \frac{\partial g}{\partial y} \\
\frac{\partial F}{\partial x} & \frac{\partial F}{\partial y}
\end{array}\right)=(2 Y+11)\left(6 X+22-\frac{22(2 Y+11)}{X^{3}}\right)+\frac{22\left(3 X^{2}+22 X+33\right)}{X^{2}} .
$$

This function has five zeroes in $E(\mathbf{R})$ and the function $t$ assumes the values $-3.60,0.34$, $-5.19,-0.44$ and 2.57 in these zeroes. Since the absolute values of these numbers exceed $\frac{1}{20}$, the zeroes are not contained in $U$ and hence $g$ assumes no extremal values on $U$.

It follows that on each of the five intervals $I$ of part (a) the function $g$ is monotonous and assumes either only positive or only negative values. This implies that on each $I$ we have $|g(P)| \geq \min \left(|g(z)|,\left|g\left(z^{\prime}\right)\right|\right)$ where $z, z^{\prime}$ are the boundary points of $I$. In our case the boundary points are given by the equation $t=Y-\frac{11}{X}= \pm \frac{1}{20}$. The values of $g$ in these points are given by $9.30,-2.05,1.63,-4.21,159.23$ (for the plus sign) and 10.18, -1.46 , $1.14,-3.39$ and 159.62 (for the minus sign) respectively. The number with the smallest absolute value is 1.14 which still exceeds 1 . This proves the Lemma.

Remark. The proof of Lemma 2.1 is related to the arguments in [16, section 2] and [15, section 2.4]. In our case the situation is relatively straightforward because all zeroes of the function $t$ are simple.

For any non-constant $f$ in the function field of $E$ and any point $P \in E(\mathbf{Q})$ we let

$$
H_{f}(P)=\prod_{p \leq \infty} \max \left(1,|f(P)|_{p}\right)
$$


denote the height of $P$ with respect to $f$. We let $h_{f}(P)=\log H_{f}(P)$ denote the logarithmic height of $P$ with respect to the function $f$. The canonical height $\widehat{h}(P)$ of $P$ is defined as $\frac{1}{\operatorname{deg} f} \lim _{n \rightarrow \infty} h_{f}\left(2^{n} P\right) / 4^{n}$. Here $f$ can be any even non-constant function on $E$, for instance $f=X$. See [12, VIII]. The function $t=Y-11 / X$ is not even. We consider the height function $h_{t}$ and compare it to the canonical height. For our purposes the following weak estimate is sufficient.

Lemma 2.2. For every point $P \in E(\mathbf{Q})$ we have

$$
\widehat{h}(P) \leq \frac{1}{3} h_{t}(P)+4.52 .
$$

Proof. Let $P=(x, y) \in E(\mathbf{Q})$. We first compare $h_{t}(P)$ to $h_{X}(P)$. For every finite prime $p$ we have

$$
\max \left(1,|x|_{p}\right) \leq \max \left(1,\left|y-\frac{11}{x}\right|_{p}\right)^{2 / 3}
$$

This is obvious when $|x|_{p} \leq 1$. When $|x|_{p}>1$, the Weierstrass equation implies that $\operatorname{ord}_{p}(x)=-2 k$ and $\operatorname{ord}_{p}(y)=-3 k$ for some $k>0$. It follows that $\operatorname{ord}_{p}\left(y-\frac{11}{x}\right)=-3 k$ and the inequality follows.

At the infinite prime we have

$$
\max (1,|x|) \leq 7 \max \left(1,\left|y-\frac{11}{x}\right|\right)^{2 / 3} .
$$

This is obvious when $|x| \leq 7$. If $|x|>7$, we observe that $E$ has no real points with $x$-coordinate less than -7 , so that we actually have $x>7$. Then we have $\left(y+\frac{11}{2}\right)^{2} \geq$ $\left(x^{3 / 2}+\frac{11}{2}\right)^{2}$ and hence $\left|y-\frac{11}{x}\right| \geq\left|y+\frac{11}{2}\right|-\left|\frac{11}{2}+\frac{11}{x}\right| \geq x^{3 / 2}+\frac{11}{2}-\frac{99}{14} \geq\left(\frac{1}{7} x\right)^{3 / 2}$.

Taking the product, it follows that $H_{X}(P) \leq 7 H_{t}(P)^{2 / 3}$ and hence

$$
h_{X}(P) \leq \frac{2}{3} h_{t}(P)+\log 7 .
$$

To conclude the proof, we compare $h_{X}(P)$ to $\widehat{h}(P)$. Since the discriminant of $E$ is $11^{3}$ and its $j$-invariant is $-2^{15}$, Silverman's estimate [11, Thm.1.1] implies $\widehat{h}(P) \leq \frac{1}{2} h_{X}(P)+3.54$. Combining the two estimates gives

$$
\widehat{h}(P) \leq \frac{1}{2}\left(\frac{2}{3} h_{t}(P)+\log 7\right)+3.54<\frac{1}{3} h_{t}(P)+4.52,
$$

as required 


\section{The proof.}

In this section we prove Theorem 1.1. Our proof closely follows the strategy of [14]. Let $\omega$ denote the invariant differential $\frac{d X}{2 Y+11}$ of $E$. We define the elliptic logarithm of a point $P \in E(\mathbf{R})$ by

$$
\lambda(P)=\int_{\infty}^{P} \omega
$$

Since $\lambda(P)$ depends on the path of integration in $E(\mathbf{C})$, it is only well defined up to the period lattice of $E$. Since $P$ is in $E(\mathbf{R})$, there is a path of integration inside the real locus $E(\mathbf{R})$. Therefore $\lambda(P)$ is equal to a real number modulo the period lattice and this real number is unique up to a multiple of the real period

$$
\Omega=\int_{c}^{\infty} \frac{d x}{\sqrt{q(x)}}=4.8024 \ldots
$$

Here $c=-6.8026 \ldots$ denotes the unique real zero of $q(x)=x^{3}+11 x^{2}+33 x+\frac{121}{4}$. It follows that $\lambda(P)$ is a well defined element of $\mathbf{R} / \Omega \mathbf{Z}$. The map $P \mapsto \lambda(P)$ is a continuous group isomorphism $E(\mathbf{R}) \longrightarrow \mathbf{R} / \Omega \mathbf{Z}$. In order to avoid ambiguity, we assume that $\lambda(P)$ is a real number satisfying $0 \leq \lambda(P)<\Omega$.

Lemma 3.1. For any cusp $Q$ of $E$ we have $\lambda(Q)=\frac{k}{11} \Omega$ for some integer $k$.

Proof. Any cusp $Q=(x, y)$ is contained in $E(\mathbf{R})$ so that $\lambda(Q)=r \Omega$ for some $r \in \mathbf{R}$. Since we have $y=11 / x$, the $x$-coordinate of $Q$ is a zero of the polynomial $p(X)=$ $X^{5}+11 X^{4}+33 X^{3}-121 X-121$. One checks that the 11-division polynomial of $E$ is divisible by $X^{5}+11 X^{4}+33 X^{3}-121 X-121$. This implies that $\lambda(Q)=\frac{k}{11} \Omega$ for some $k \in \mathbf{Z}$, as required.

Alternatively, one can avoid the computation of the 11-division polynomial and proceed as follows. The curve $E$ admits complex multiplication by the ring $\mathbf{Z}\left[\frac{1+\sqrt{-11}}{2}\right]$ and the kernel of the endomorphism $\sqrt{-11}$ is precisely the order 11 group $G$ generated by $\lambda^{-1}\left(\frac{1}{11} \Omega\right)$. Since the Galois group of $\overline{\mathbf{Q}}$ over $\mathbf{Q}$ preserves $G$, there is a unique monic degree 5 polynomial $q(X) \in \mathbf{Q}[X]$ whose zeroes are precisely the $x$-coordinates of the points of $G$. By the Nagell-Lutz Theorem [12, VII.3.4], each point $(x, y) \in G$ has the property that $11 x$ is an algebraic integer. Therefore we can compute $q(X)$ by calculating sufficiently accurate approximations to its roots. We find that $p(X)=q(X)$ and hence $Q \in G$. This proves the lemma.

Finally, the lemma also follows from the fact that Halberstadt's isomorphism [6, 3.3] is known to map the cusps of the modular curve $X_{n s}(11)$ to certain 11-torsion points of $E$. See section 4 .

Proof of Theorem 1.1. First we check that the only integers $k$ with $|k| \leq 20$ for which there are points $P=(x, y)$ in $E(\mathbf{Q})$ with $x /(x y-11)$ equal to $k$ are $k=0, \pm 2,-6$ and -8 . These values of $k$ already account for the seven points listed in Theorem 1.1. To prove the theorem, let $P=(x, y)$ be a point in $E(\mathbf{Q})$ for which $|x /(x y-11)|$ is an integer exceeding 20. Since $t=Y-11 / X$, we have $|t(P)|<\frac{1}{20}$. By Lemma 2.1 there is a cusp $Q$ and an open interval $I \subset U$ containing both $P$ and $Q$. 
Let $\int_{Q}^{P} \omega$ denote the integral from $Q$ to $P$ of the invariant differential $\omega$ along a path inside the interval $I$. Then $\int_{Q}^{P} \omega$ is real and we estimate its absolute value. Writing $F(X, Y)=Y^{2}+11 Y-X^{3}-11 X^{2}-33 X$, we have for every function $f$ on $E$ that

$$
d f=\operatorname{det}\left(\begin{array}{ll}
\frac{\partial f}{\partial X} & \frac{\partial f}{\partial Y} \\
\frac{\partial F}{\partial X} & \frac{\partial F}{\partial Y}
\end{array}\right) \omega .
$$

In particular, taking $f$ equal to $t$, we find that $d t / \omega$ is equal to the function $g=3 X^{2}+$ $22 X+33+\frac{11(2 Y+11)}{X^{2}}$ of Lemma 2.1. Therefore

$$
\int_{Q}^{P} \omega=\int_{0}^{t(P)} \frac{\omega}{d t} d t=\int_{0}^{t(P)} \frac{d t}{g} .
$$

By Lemma 2.1 we have $|g(x, y)| \geq 1$ for all $(x, y) \in I$. Therefore we have $\left|\int_{Q}^{P} \omega\right| \leq$ $\left|\int_{0}^{t(P)} d t\right|=|t(P)|$. Since $\lambda(P)-\lambda(Q) \equiv \int_{Q}^{P} \omega$ modulo $\Omega \mathbf{Z}$, there exists $n^{\prime} \in \mathbf{Z}$ such that

$$
\left|\lambda(P)-\lambda(Q)+n^{\prime} \Omega\right| \leq|t(P)|
$$

By Lemma 3.1 we then have

$$
\left|n \frac{\Omega}{11}-\lambda(P)\right| \leq|t(P)|, \quad \text { for some } n \in \mathbf{Z}
$$

Since $1 / t(P)$ is in $\mathbf{Z}$, we have that $h_{t}(P)=-\log |t(P)|$. Therefore Lemma 2.2 implies that

$$
\left|n \frac{\Omega}{11}-\lambda(P)\right|<\exp (13.56-3 \widehat{h}(P)), \quad \text { for some } n \in \mathbf{Z} \text {. }
$$

We write $P_{0}$ for the generator $(0,0)$ of the group $E(\mathbf{Q})$ so that $P=m P_{0}$ for some integer $m$. Since $\widehat{h}(P)=m^{2} \widehat{h}\left(P_{0}\right)$ and $\widehat{h}\left(P_{0}\right)=0.04489 \ldots$ this gives

$$
\left|n \Omega-m \lambda\left(11 P_{0}\right)\right| \leq 11 \cdot \exp \left(13.56-0.13 \cdot m^{2}\right), \quad \text { for some } n \in \mathbf{Z} .
$$

On the other hand, since $P_{0}$ is not a torsion point, $n \Omega-m \lambda\left(11 P_{0}\right)$ is a non-vanishing linear form in the elliptic logarithms $\Omega$ and $\lambda\left(11 P_{0}\right)$. We recall the explicit lower bound that Sinnou David obtained for such forms [5, Théorème 2.1]. In David's notation we have $K=\mathbf{Q}, D=1$ and $k=2$. The coefficients $\beta_{0}, \beta_{1}, \beta_{2}$ of his linear form are equal to $0, n,-m$ in our case. We have $u_{1}=\Omega$ with $\gamma_{1}$ equal to the point at infinity and $u_{2}=\lambda\left(11 P_{0}\right)$ with $\gamma_{2}=11 P_{0}$. It follows that David's constants $V_{1}$ and $V_{2}$ are given by $V_{1}=1.415 \ldots \times 10^{27}$ and $V_{2}=7.98 \ldots \times 10^{14}$.

David's estimates imply that when $B=\max (|m|,|n|)$ exceeds $V_{1}=1.415 \ldots \cdot 10^{27}$, then we have

$$
\left|n \Omega-m \lambda\left(11 P_{0}\right)\right|>\exp \left(-7.658 \times 10^{44}(\log B+1)(\log \log B+15 \log (2)+1)^{3}\right) .
$$


We consider first the case $|m| \geq 12$. Then the right hand side of inequality $(1)$ is $<0.07$. Since $\Omega=4.8024 \ldots$ and $\lambda\left(11 P_{0}\right)=3.5579 \ldots$, this easily implies that $|m| \geq|n|$ and hence $B=|m|$. We claim that

$$
|m|<1.415 \times 10^{27}
$$

Indeed, if the inequality is false, we may apply David's lower bound (2). Comparing the inequalities (1) and (2) one finds $|m|<3.62 \times 10^{25}$, contradicting our assumption.

The bound on $|m|$ is very large. However, we can use it to obtain a better bound by observing that for $|m| \geq 12$ the right hand side of (1) is less than $0.4 \frac{\Omega}{|m|}$. This leads to the inequality

$$
\left|\frac{n}{m}-\frac{\lambda\left(11 P_{0}\right)}{\Omega}\right|<\frac{0.4}{m^{2}}<\frac{1}{2 m^{2}}
$$

implying that $n / m$ is a convergent $p_{k} / q_{k}$ of the continued fraction expansion of $\lambda\left(11 P_{0}\right) / \Omega$. By (3) we must have that $q_{k}<1.415 \times 10^{27}$. Using Zagier's algorithm [17] we compute $\lambda\left(11 P_{0}\right)$ and $\Omega$ with an accuracy of 60 decimal digits and verify that for $k>55$ the convergents $p_{k} / q_{k}$ do not satisfy $q_{k}<1.415 \times 10^{27}$. Note that replacing $\lambda\left(11 P_{0}\right) / \Omega$ by its approximation $\sigma$ to 60 decimal digits, does not affect the first 55 convergents. This follows from the inequality

$$
\left|\frac{n}{m}-\sigma\right| \leq\left|\frac{n}{m}-\frac{\lambda\left(11 P_{0}\right)}{\Omega}\right|+\left|\frac{\lambda\left(11 P_{0}\right)}{\Omega}-\sigma\right|<\frac{0.4}{m^{2}}+10^{-60}<\frac{1}{2 m^{2}} .
$$

On the other hand, one checks that for $k \leq 55$ inequality (1) does not hold when $q_{k} \geq 12$. Indeed, one has

$$
\left|p_{k} \Omega-q_{k} \lambda\left(11 P_{0}\right)\right|>11 \cdot \exp \left(13.56-0.13 \cdot q_{k}^{2}\right)
$$

for all $k \leq 55$ for which $q_{k} \geq 12$. This contradicts our assumption that $|m| \geq 12$.

It remains to deal with the case $|m|<12$. Inspection of the points $m P_{0}$ for $-12<$ $m<12$ shows that only for $m=-2,-1,0,1,2,3$ and 4 the point $(x, y)=m P_{0}$ has the property that $x /(x y-11)$ is integral. In fact, these are the seven points $(-2,-6),(0,-11)$, $\infty,(0,0),(-2,-5),(-11 / 4,-33 / 8)$ and $(-6,-2)$ respectively. So once again we recover the seven points of Theorem 1.1. This completes the proof of Theorem 1.1.

The continued fraction argument to reduce the upper bound for $|m|$ is particularly simple in our case because the rank of the Mordell-Weil group of $E$ is 1 . In general, one employs a lattice reduction algorithm that can handle lattices of higher rank. See [16] where the LLL algorithm is used.

Normalizations. Our definition of the canonical height agrees with the one given by Silverman [12, VIII]. The canonical height used by the PARI and MAGMA programs is twice as large, while the canonical height used by Sinnou David [5] is three times ours. In a similar way, our definition of the real period $\Omega$ agrees with the one given by Silverman [12] and the one used by PARI. The one used by Zagier [17] is twice as large. 


\section{The modular curve.}

Let $X_{n s}(11)$ denote the modular curve associated to the normalizer of a non-split Cartan subgroup of level 11. It parametrizes elliptic curves with a certain level 11 structure $[10$, Appendix]. In 1977, G. Ligozat [9, Proposition 4.3.8.1] showed that $X_{n s}(11)$ is isomorphic to the genus 1 curve given by the Weierstrass equation $Y^{2}+Y=X^{3}-X^{2}-7 X+10$. Replacing $X$ by $X+4$ and $Y$ by $Y+5$, we see that this curve is isomorphic to the curve $E$ given by

$$
Y^{2}+11 Y=X^{3}+11 X^{2}+33 X .
$$

In this section we show that the $j$-invariant of an elliptic curve parametrized by a point $P=(x, y) \in E(\mathbf{Q})$ is in $\mathbf{Z}$ if and only if $x /(x y-11) \in \mathbf{Z}$. This shows that Theorem 1.2 follows from Theorem 1.1.

The curve $X_{n s}(11)$ admits a natural morphism $j: X_{n s}(11) \longrightarrow \mathbf{P}^{1}$, mapping a point $P$ of $X_{n s}(11)$ to the $j$-invariant of the elliptic curve parametrized by $P$. The morphism $j$ has degree 55 and is defined over $\mathbf{Q}$. The formula for the natural morphism from $E$ to the $j$-line depends on the choice of an isomorphism between the modular curve $X_{n s}(11)$ and the elliptic curve $E$. Since translation by a rational point is a $\mathbf{Q}$-rational automorphism of $E$, there are infinitely many such choices. We follow Halberstadt [6] and choose one of the two isomorphisms that map the five cusps of $X_{n s}(11)$ to the zeroes of the degree 5 function $t=Y-11 / X$. See [6, section 3]. For formulas that are based on a different choice see [3]. According to Halberstadt's explicit formula, we have

$$
j(X, Y)=\frac{h(X, Y)}{(X Y-11)^{11}},
$$

where $h(X, Y)$ is equal to

$$
\left(X^{2}+11 X+22\right)^{3}\left(\left(11 X^{2}+88 X+121\right) Y+2 X^{4}+55 X^{3}+451 X^{2}+1452 X+1452\right)^{3} g(X, Y)
$$

and $g(X, Y)$ is the polynomial

$$
\begin{aligned}
& \left(6750 X^{8}+337590 X^{7}+5159935 X^{6}+36807958 X^{5}+145636931 X^{4}+341425458 X^{3}\right. \\
& \left.+474292533 X^{2}+362189058 X+117523307\right) Y+51975 X^{9}+1746052 X^{8}+24440064 X^{7} \\
& +188870352 X^{6}+892661770 X^{5}+2692703508 X^{4}+5217583888 X^{3}+6299026712 X^{2} \\
& +4320837279 X+1288408000 .
\end{aligned}
$$

Our formula follows from Halberstadt's formula $[6,(2-1)]$ by dividing his polynomial $f_{3}^{3} f_{4}^{3}$ by $f_{5}^{2}$. After replacing $X$ by $X+4$ and $Y$ by $Y+5$, the quotient is our polynomial $g(X, Y)$.

One checks that the seven points listed in Theorem 1.1 give rise to the $j$-invariants $-5280^{3}, 66^{3}, 12^{3},-3 \cdot 160^{3},-640320^{3}, 0$ and $2 \cdot 30^{3}$ respectively. These are precisely the $j$-invariants of the elliptic curves with complex multiplication by the quadratic orders of discriminant $-67,-16,-4,-27,-163,-3$ and -12 respectively.

Theorem 4.1. Let $(x, y)$ be a rational point on the elliptic curve $E$ given by the Weierstrass equation $Y^{2}+11 Y=X^{3}+11 X^{2}+33 X$. Then $j(x, y)$ is in $\mathbf{Z}$ if and only if $x /(x y-11)$ is in $\mathbf{Z}$.

Proof. We study integrality of $j(x, y)$ and $x /(x y-11)$ one prime $l$ at a time. It follows from the Weierstrass equation that we can write $x=r / t^{2}$ and $y=s / t^{3}$ for certain $r, s, t \in \mathbf{Z}$ 
satisfying $\operatorname{gcd}(r s, t)=1$. The denominators of both $j(x, y)=h(x, y) /(x y-11)^{11}$ and $x /(x y-11)$ divide a power of $r s-11 t^{5}$. Therefore, if $l$ is a prime not dividing $r s-11 t^{5}$, both $j(x, y)$ and $x /(x y-11)$ are integral at $l$. Let therefore $l$ be a prime that divides $r s-11 t^{5}$. If $l$ divides $t$, then it divides $r s$, which it cannot. So $l$ does not divide $t$. This implies that both $x$ and $y$ are $l$-integral and $l$ divides $x y-11$.

Suppose $l \neq 11$. Then $l$ does not divide $x$, so that $x /(x y-11)$ is not integral at $l$. Substituting $Y=11 / X$ in the Weierstrass equation we find that $l$ divides $p(x)$ where $p(X)=X^{5}+11 X^{4}+33 X^{3}-121 X-121$. Suppose now that $j(x, y)=h(x, y) /(x y-11)^{11}$ is integral at $l$. Then $l$ divides $h(x, y)$. Substituting $Y=11 / X$ in the polynomial $h(X, Y)$ and multiplying by $X^{4}$, we find that $l$ divides $r(x)$ where $r(X)$ is a certain degree 31 polynomial in $X$ with integral coefficients. Therefore $l$ divides the resultant of $p(X)$ and $r(X)$, which one checks to be equal to $11^{63}$. This shows that $l=11$. This contradicts our assumption and we conclude that $j(x, y)$ is not integral at $l$.

Finally, suppose $l=11$. Since $l$ divides $x y-11$, it also divides $x y$ and it follows from the Weierstrass equation that 11 actually divides both $x$ and $y$. It follows that 11 divides $x y-11$ exactly once so that $x /(x y-11)$ is integral at 11 . To see that $j(x, y)=h(x, y) /(x y-11)^{11}$ is also integral at 11 , we observe that the exact power of 11 dividing $(x y-11)^{11}$ is $11^{11}$. On the other hand, one checks that when both $x$ and $y$ are divisible by 11 , the numerator $h(x, y)$ is divisible by $11^{14}$. Therefore $h(x, y) /(x y-11)^{11}$ is divisible by $11^{3}$ and hence $j(x, y)$ is certainly integral at 11 .

This proves the Theorem.

\section{Bibliography}

[1] Baker, A.: A remark on the class number of quadratic fields Bull. London Math. Soc. 1 (1969), 98-102.

[2] Baran, B.: A modular curve of level 9 and the class number one problem, Journal of Number Theory 129 (2009), 715-728.

[3] Chen, I. and Cummins, C.: Elliptic curves with non-split mod 11 representations, Math Comp. 73 (2004), 869-880.

[4] Cremona, J.: Algorithms for modular elliptic curves, 2nd ed, Cambridge University Press, Cambridge 1997.

[5] David, S.: Minorations de formes linéaires de logarithmes elliptiques, Mémoires Soc. Math. France (N.S) 62 (1995).

[6] Halberstadt, E.: Sur la courbe modulaire $X_{\text {ndep }}(11)$, Experimental Math., 7 (1998), 163-174.

[7] Heegner, K.: Diophantische Analysis und Modulfunktionen, Math. Zeit. 59 (1952), 227-253.

[8] Kenku, M.A.: A note on the integral points of a modular curve of level 7, Mathematika 32 (1985), 45-48.

[9] Ligozat, G.: Courbes modulaires de niveau 11, in J-P. Serre and D.B. Zagier Eds, Modular Functions of one variable V, LNM 601, 149-237, Springer-Verlag, 1977

[10] Serre, J-P.: Lectures on the Mordell-Weil Theorem, Aspects of Mathematics 15, Vieweg, Braunschweig 1997.

[11] Silverman, J.: The difference between the Weil height and the canonical height on elliptic curves, Math. Comp. 55 (1990), 723-743. 
[12] Silverman, J.: The arithmetic of elliptic curves, 2nd ed, Graduate Texts in Mathematics 106, Springer-Verlag, New York 2009.

[13] Stark, H.M.: On complex quadratic fields with class number equal to one, Trans. Amer. Math. Soc. 122 (1966), 112-119.

[14] Stroeker, R.J. and Tzanakis, N.: Solving elliptic diophantine equations by estimating linear forms in elliptic logarithms, Acta Arith. 67 (1994), 177-196.

[15] Stroeker, R.J. and Tzanakis, N.: Computing all integer solutions of a genus 1 equation, Math. Comp. 72 (2003) 1917-1933.

[16] Tzanakis, N.: Solving elliptic diophantine equations by estimating linear forms in elliptic logarithms. The case of quartic equations, Acta Arith. 75 (1996), 165-190.

[17] Zagier, D.: Large integral points on elliptic curves, Math. Comp. 48 (1987), 425-436.

René Schoof

Università di Rome "Tor Vergata"

Dipartimento di Matematica

I-00133 Roma, Italy

schoof@mat.uniroma2.it
Nikos Tzanakis

Department of Mathematics University of Crete

Iraklion, Greece

tzanakis@math.uoc.gr 